\title{
Drag forces in the near and distant solar system
}

\author{
Klaus Scherer ${ }^{1}$ and Hans-Jörg Fahr ${ }^{2}$ \\ ${ }^{1}$ Max-Planck-Institut für Aeronomie, 37191 Katlenburg-Lindau, Germany \\ ${ }^{2}$ Institut für Astrophysik und extraterrestrische Forschung der Universität Bonn, Auf dem Hügel 71, 53121 Bonn, Germany
}

(Received October 7, 1997; Revised December 19, 1997; Accepted January 7, 1998)

\begin{abstract}
Like the solar photons the solar wind particles induce a drag force onto the zodiacal dust grains in the heliosphere. For the distant solar wind with high Mach numbers the drag coefficient is a constant, but close to the Sun, where Mach numbers become small, the drag coefficient is a complicated function of the ion sound speed, density and temperature. We discuss the dynamics of dust particles due to this drag force and compare it with that in the distant solar wind. Especially in the near solar wind the eccentricity varies in a complicated way with the inclination of the orbits, also the semimajor axis decreases faster closer to the Sun. These variations are quite different in the distant solar wind.

In addition, we apply an analogous mathematical formalism to the dust dynamics in the outer region of the heliosphere (>20 AU) where the neutral gas density becomes comparable or larger than that of the solar wind plasma. Here the neutral hydrogen gas induces a drag force onto the dust particles similar to the plasma PoyntingRobertson effect. But different to the radial solar wind, the velocity of the interstellar gas is mono-directional, and hence with respect to the inflow direction of the interstellar material this introduces an axial-symmetric force onto the dust particles. This force acts asymmetric in the orbit, and causes the eccentricity to increase fairly fast. The lifetime for dust grains in the Edgeworth-Kuiper Belt is no longer determined by the electromagnetic Poynting-Robertson lifetime, but by that of the neutral gas and is in the order of half a million years for a $10 \mu \mathrm{m}$ sized particle.
\end{abstract}

\section{Introduction}

As it was shown (by Banaszkiewicz et al., 1994; Fahr et al., 1995) in the "asymptotic" solar wind (Mach numbers $M \geq 10$ ) the plasma drag forces are of the same order as the electromagnetic Poynting-Robertson force. Not to be confused with "fast" and "slow" solar wind, we introduce the notation of the "distant" and "near" solar wind, with the meaning that the distant solar wind has evolved to high Mach numbers $M \gg 1$, while the region close to the corona, where the Mach numbers are of the order $M \approx 1$ or less is called the near solar wind. Previously it was shown (Banaszkiewicz et al., 1994) for the distant solar wind that the drag coefficient reduces to a constant. With this constant the variation of the classical elements can be analytically average over one orbit, which allows for a fast calculation of the evolution of the orbital elements and the number density distribution (Banaszkiewicz et al., 1994; Gor'kavyi et al., 1997).

Because the near solar wind strongly varies with solar distance and solar latitude, and the Mach numbers are low it is impossible to find analytical solutions. On the other hand the knowledge of the dynamical behaviour of the dust grains close to the Sun elucidates how the solar wind evolves, especially in the ecliptic plane. Moreover, due to strong variations of the solar wind speed over the poles and in the ecliptic, the dynamical behaviour of the dust particles is quite different to the distant solar wind. At this stage of the work it is not clear if one can explain the spherical component of

Copy right (C) The Society of Geomagnetism and Earth, Planetary and Space Sciences (SGEPSS); The Seismological Society of Japan; The Volcanological Society of Japan; The Geodetic Society of Japan; The Japanese Society for Planetary Sciences. the Zodiacal Light close to the Sun by the kind of plasma interactions. The reason is that we have chosen a semianalytical three-dimensional model of the near solar wind, in which we did not self-consistently calculate the temperature and density variation, which is in principle possible and will be done in the future.

In the Outer Heliosphere the neutral gas density becomes comparable with or larger than the solar wind density. Therefore, the drag force induced by the neutral hydrogen gas should become comparable to the plasma PoyntingRobertson force. The mathematical formulation of that problem is analogous to that in the case of the plasma and all results can be used. One needs only carefully to reinterpret the physical properties. One crucial difference of the neutral drag force is its non-radial character compared to the radial one of the electromagnetic and plasma force. This dramatically reduces the lifetime of the Edgeworth-Kuiper Belt dust grains.

In Section 1 we give a short description of the derivation of the plasma Poynting-Robertson force and the indirect force, followed in Section 2 by a discussion of the dynamics in the near solar wind regime. In Section 3 we apply the drag force formalism to the action of the interstellar neutral hydrogen atoms penetrating into the heliosphere.

\section{The Drag Force}

The total force acting on an orbiting dust grain consists of the contribution of the solar gravity $\vec{F}_{\mathrm{K}}$, the gravitational perturbation force by the planets $\vec{F}_{\mathrm{P}}=\sum_{i} \vec{F}_{p_{i}}$, where the index $i$ denotes the planet (esimal), the electromagnetic PoyntingRobertson force $\vec{F}_{\mathrm{e}}$, the corpuscular drag forces $\vec{F}_{\mathrm{D}}$, the 
Lorentz force $\vec{F}_{\mathrm{L}}$, general relativistic effects $\vec{F}_{\mathrm{R}}$ and others (Scherer et al., 1996). Here we will concentrate on the corpuscular drag forces, which can be decomposed into:

$$
\vec{F}_{\mathrm{D}}=\vec{F}_{\mathrm{IF}}+\sum_{x} \vec{F}_{x}
$$

where the index $x$ describes the species of the impacting particle $(x=p, \alpha, H, \ldots)$. A study of all these forces would go far beyond the scope of this paper. In forthcoming publications we will study the different forces near the Sun and in the outer heliosphere in more detail, here we restrict ourselves onto the drag forces which is dominant for spherical particles larger than $\gtrsim 1 \mu \mathrm{m}$. The corpuscular drag force is proportional to the geometric cross section $A=\pi s^{2}$ of a dust grain with radius $s$ and the modulus of the relative velocity $v_{\text {rel }}$ between the plasma ion and the dust particle. The relevant type of the collision is described by the adsorption or sticking factor $1 \leq \xi \leq 2$ (i.e. specular $(\xi=1)$, diffuse $(\xi=1)$, or adsorption $(\xi>1))$, which describes the momentum transfer $\xi m_{x} \vec{v}_{\text {rel }}$ per impact. Here $m_{x}$ is the mass of the particle of species $x$. Finally, the force is given by:

$$
\vec{F}_{x}\left(\vec{v}_{\text {rel }}\right)=\xi \int\left[f\left(\vec{v}_{\text {rel }}, \vec{r}\right) A v_{\text {rel }}\right] m_{x} \vec{v}_{\text {rel }} d^{3} v
$$

(Banaszkiewicz et al., 1994). The term inside the square bracket is the collision frequency in the velocity space, and $f(\vec{v}, \vec{r})$ is the normalized distribution function of the respective species. Banaszkiewicz et al. (1994) and Fahr et al. (1995) assumed that the distribution function can be represented by a shifted mono-Maxwellian distribution:

$$
f(\vec{v}, \vec{r})=\frac{n(\vec{r})}{\sqrt{\pi^{3}} c_{x}^{3}} \exp \left(-\frac{\left(\vec{v}_{\mathrm{sw}}-\vec{v}\right)^{2}}{c_{x}^{2}}\right)
$$

where $v, v_{\text {sw }}$, and $c_{x}$ are the speed of the individual solar wind particle, the solar wind bulk velocity and the sound speed of the species $x$. After integration the force per unit mass yields

$$
\vec{F}=-\pi n(\vec{r}) \frac{m_{x}}{m_{\mathrm{D}}} s^{2} u^{2} c_{\mathrm{D}} \vec{e}_{v_{\mathrm{u}}}=-\gamma_{\mathrm{sw}} \vec{e}_{\mathrm{u}}
$$

where $m_{\mathrm{D}}$ is the mass of the dust grain and the drag coefficient $c_{\mathrm{D}}$ is given by:

$$
\begin{aligned}
c_{\mathrm{D}}=\frac{\xi}{M} & {\left[\left(1+\frac{1}{2 M^{2}}\right) \frac{e^{-M^{2}}}{\sqrt{\pi}}\right.} \\
& \left.+M\left(1+\frac{1}{M^{2}}-\frac{1}{4 M^{4}} \operatorname{erf}(M)\right)\right]
\end{aligned}
$$

where $M$ is the Mach number $M=u / c_{x}, \vec{u}$ is the velocity of the dust grain relative to the solar wind speed.

In the solar system (except planetary environments) one needs not to take into account collective effects of the impinging ions, because the Debye-length of the solar wind plasma is much larger than the grain radius (i.e. Northrop, 1992). In addition, the net drag force induced by the Coulomb interaction between the charged dust particle and the plasma, the so-called indirect force $F_{\mathrm{IF}}$, (Northrop and Birmingham, 1990) had not to be taken into account. This force (in c.g.s units) for a Maxwellian plasma is described by:

$$
\begin{aligned}
F_{\mathrm{IF}}= & \frac{8 \sqrt{\pi}(Z e)^{2} e^{2} n_{\mathrm{sw}}}{m_{\mathrm{sw}} u^{2}}\left(1+\frac{m_{\mathrm{sw}}}{m_{\mathrm{D}}}\right) \\
& \cdot\left[-M e^{-M^{2}}+\frac{\sqrt{\pi}}{2} \operatorname{erf}(M)\right] \ln \frac{\lambda_{D}}{s}
\end{aligned}
$$

where $e$ is the elementary charge, $Z e$ is the grain charge, $n_{\mathrm{sw}}$ is the solar wind proton density, $m_{\mathrm{sw}}$ is the mass of the solar wind ions (protons), $\lambda_{\mathrm{D}}$ is the Debye length, and $s$ is the grain radius. The ratio of the plasma Poynting-Robertson force to the indirect force is:

$$
\begin{aligned}
\frac{F_{\mathrm{PPR}}}{F_{\mathrm{IF}}} \approx & \frac{\sqrt{\pi} m_{\mathrm{sw}}^{2} u^{4} s^{2}}{8 e^{4} Z^{2} \ln \left(\frac{\lambda_{\mathrm{D}}}{s}\right)} \\
\approx & 10^{4}\left(\frac{u}{100 \mathrm{~km} \mathrm{~s}^{-1}}\right)^{4}\left(\frac{s}{10 \mu \mathrm{m}}\right)^{2} \\
& \cdot\left(\frac{10^{3}}{Z}\right)^{2}\left(\frac{20}{\ln \left(\frac{\lambda_{\mathrm{D}}}{s}\right)}\right)
\end{aligned}
$$

Thus, the indirect force is very weak compared to the direct plasma drag forces, and even does not play a role very close to the Sun $\left(\ll 2 R_{\odot}\right)$ where the solar wind is subsonic, because the relative velocity between the Keplerian motion of a dust grain (about $400 \mathrm{~km} \mathrm{~s}^{-1}$ at $2 R_{\odot}$ ) and solar wind is still large. This is caused by the fact, that the solar wind has mainly a radial velocity component, while the orbital velocity $\vec{v}_{\mathrm{D}}$ of the dust grain has mainly an azimuthal component, and thus the modulus of $u$ is larger than that of the individual velocities $\vec{v}_{\mathrm{sw}}, \vec{v}_{\mathrm{D}}$. On the other hand, the Mach number still substantly decreases, because of the increase in temperature, but remains $\gg 0$. Hence we can safely neglect the indirect force.

As was shown in Banaszkiewicz et al. (1994) the corpuscular force is of the same order as the electromagnetic PoyntingRobertson force, where in both cases the dust grains are treated as spheres. If one wants to follow the trajectory of an individual dust grain one has to take into account: 1) that both forces depend on the shape and on the optical properties of the dust grain; 2) that the solar wind is highly variable on short timescales; and 3) that the dust grain most probably rotates which in turn influences the first point. To avoid these complications, we averaged over one orbit of a dust grain, which smears out the shape of the rotating dust grain almost to a sphere. This averaging procedure eventually leads to an averaged force equation, which is equal to the evolution of a spherical dust grain with a given radius $s$ and density $\rho$. In the following we therefore treat the dust grains as perfect spheres with a given density. This simplification will not change the dynamics of an ensemble of dust grains, but gain a lot of computation time.

\subsection{The near solar wind}

When the dust particle spirals toward the Sun, it eventually enters the region, where the ecliptic solar wind becomes only marginally supersonic or even subsonic. In that case one cannot apply the above given formula, because the drag coefficient is no longer constant, and in that region the sound velocity changes due to the changing temperature, and the number density decreases no longer with a simple $1 / r^{2}$ law 
where $r$ is the heliocentric distance (Muhleman and Anderson, 1981; Bougeret et al., 1984).

To handle these difficulties we use the three-dimensional coronal solar wind model developed by Fahr et al. (1998) which is a polar axial symmetric and analytic model. In this model the latitudinal dependence of the asymptotic solar wind velocity is given by:

$$
v_{\mathrm{sw}_{\infty}}=v_{\mathrm{sw}_{s}}+\frac{v_{\mathrm{sw}_{s}}-v_{\mathrm{sw}_{f}}}{2}\left[1+\tanh \left(\alpha\left[\frac{z}{r}-\frac{z_{\mathrm{c}}}{r_{\mathrm{c}}}\right]\right)\right]
$$

where $v_{\mathrm{sw}_{s}}, v_{\mathrm{sw}_{f}}$ are values for the typical slow and fast solar wind velocity, $r, z$ are cylindrical coordinates $(r=$ $\left.\sqrt{x^{2}+z^{2}}\right), \alpha$ is called the "rapidity factor", and $z_{\mathrm{c}} / r_{\mathrm{c}}$ is the critical cosine for the change from the slow to fast solar winds. The velocity variation along a streamline is given as a function of the streamline element $S$ by:

$$
v_{\mathrm{sw}}(S)=\frac{v_{\mathrm{sw}_{\infty}}\left(S_{\infty}\right)}{2}\left[1+\tanh \left(\beta\left[\frac{S}{S_{\mathrm{c}}}-1\right]\right)\right]
$$

where $\beta$ is described as "steepness factor" and $S_{\mathrm{c}}$ is the critical sonic point (Fig. 1). These formulas are still too complicated to allow for a fully analytic description of the orbital evolution. Therefore, we calculate the orbital averages of the perturbations numerically. This in addition has the advantage that no approximations are necessary and also retrograde orbits can be taken into account. As can be seen from Fig. 2 the shape of the eccentricity curve changes with inclination. The reason is that for high inclinations the dust grain has to pass from the high speed polar wind through the ecliptic, where the solar wind speed is dramatically reduced. The overall evolution of the dust grain axis is very similar to that discussed for the fast solar wind (Banaszkiewicz et al., 1994) and similar the inclination, does not change, because the drag force does not induce a normal component. The different inclinations are important because of the latitudinal dependence of the solar wind.

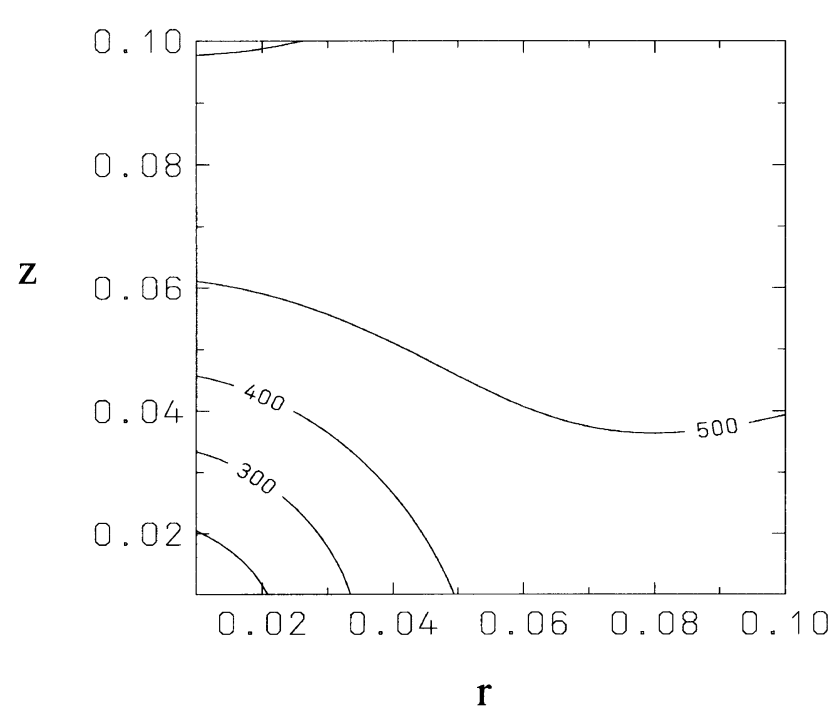

Fig. 1. The near solar wind model with $\alpha=\beta=1$, the $z$-axis points to the solar pole, and the $x$-axis is the radial distance in the ecliptic. Both are measured in AU.
About $3-4 R_{\odot}$ a slight change in the slope of the semimajor axis can be observed. This is very likely when the dust grain is orbiting inside the sonic point of the solar wind. But for a firm conclusion we need to incorporate the temperature and number density in a self consistent way. This will be done in a future publication. Nevertheless, our first results show that the dynamics close to the Sun are different compared to the distant solar wind. Much easier to handle is the neutral Poynting-Robertson drag force, which is dominant in the outer solar system, as will be shown in the next section.

\subsection{The neutral gas}

The drag force derived in Eq. (4) is independent of the charge of the projectiles, and therefore the same mathematical formalism can be applied to the momentum transfer by neutral hydrogen particles $(\mathrm{H})$. This situation becomes relevant in the outer heliosphere, beyond $20 \mathrm{AU}$ where the neutral hydrogen density becomes comparable to or larger than the solar wind density. The neutral gas is penetrating to the inner heliosphere due to the relative motion of the Sun (Osterbart and Fahr, 1992). But, instead of a radial outflowing solar wind, the drag introduced by the neutral gas in view of the low orbital dust velocities is now mono-directional, and thus acts in a different way.

One has to be careful with the distribution function $f\left(\vec{r}, \vec{v}_{\text {rel }}\right)$ of the neutral gas which is a complicated function of position and velocity, because of the ionisation effects by photoionisation, electron impact, or charge exchange processes (Bzowski et al., 1997). On the other hand, in the outer heliosphere this dependence is weak (Osterbart and Fahr, 1992; Rucinski and Bzowski, 1995; Rucinski et al., 1996; Kausch and Fahr, 1997). Therefore, we assume here that the neutral gas distribution is well described by a shifted mono-Maxwellian:

$$
f\left(\vec{r}, \vec{v}_{\mathrm{rel}}\right)=n_{\mathrm{H}} \sqrt{\left(\frac{m_{\mathrm{H}}}{2 \pi k T}\right)^{3}} \exp \left(-\frac{m_{\mathrm{H}}\left(\vec{v}-\vec{v}_{\mathrm{H}}\right)^{2}}{2 k T}\right)
$$

and the force per unit mass $\vec{F}_{\mathrm{H}}$ is:

$$
\vec{F}_{\mathrm{H}}=-\gamma_{\mathrm{H}} \vec{e}_{\mathrm{u}}
$$

where $\vec{e}_{\mathrm{u}}$ is the unit vector into the direction of the relative velocity $\vec{u}=\vec{v}_{\mathrm{H}}-\vec{v}$ between the neutral gas velocity $\vec{v}_{\mathrm{H}}$ and the orbital velocity $\vec{v}$ of the dust particle and with the short hand notation:

$$
\gamma_{\mathrm{H}}=n_{\mathrm{H}} \frac{m_{\mathrm{H}}}{m_{\mathrm{D}}} \pi s^{2} c_{\mathrm{D}} u^{2} .
$$

The Sun is moving relative to the interstellar matter with a velocity of 20-30 $\mathrm{km} \mathrm{s}^{-1}$ (for a recent review see Fahr (1996), Geiss and Witte (1996), Frisch (1995)), and the neutral gas is streaming in the opposite direction with the same velocity. We assume that the direction of the velocity vector is strictly along the $y$-axis and that the velocity distribution of the neutrals is Maxwellian with a temperature of $8000^{\circ} \mathrm{K}$ and a bulk velocity of $v_{\mathrm{H}}=26 \mathrm{~km} \mathrm{~s}^{-1}$ (Scherer et al., 1997). The sound velocity for neutral hydrogen is $c_{\mathrm{H}}=11 \mathrm{~km} \mathrm{~s}^{-1}$, and hence the drag coefficient is almost constant $(M \approx 2.4)$. Because the variations of the neutral gas density $n_{\mathrm{H}}$ beyond $20 \mathrm{AU}$ are small, it can be taken as constant with $n_{\mathrm{H}}=0.05 \mathrm{~cm}^{-3}$ (Scherer et al., 1997). With the help of Eq. (12) we then 


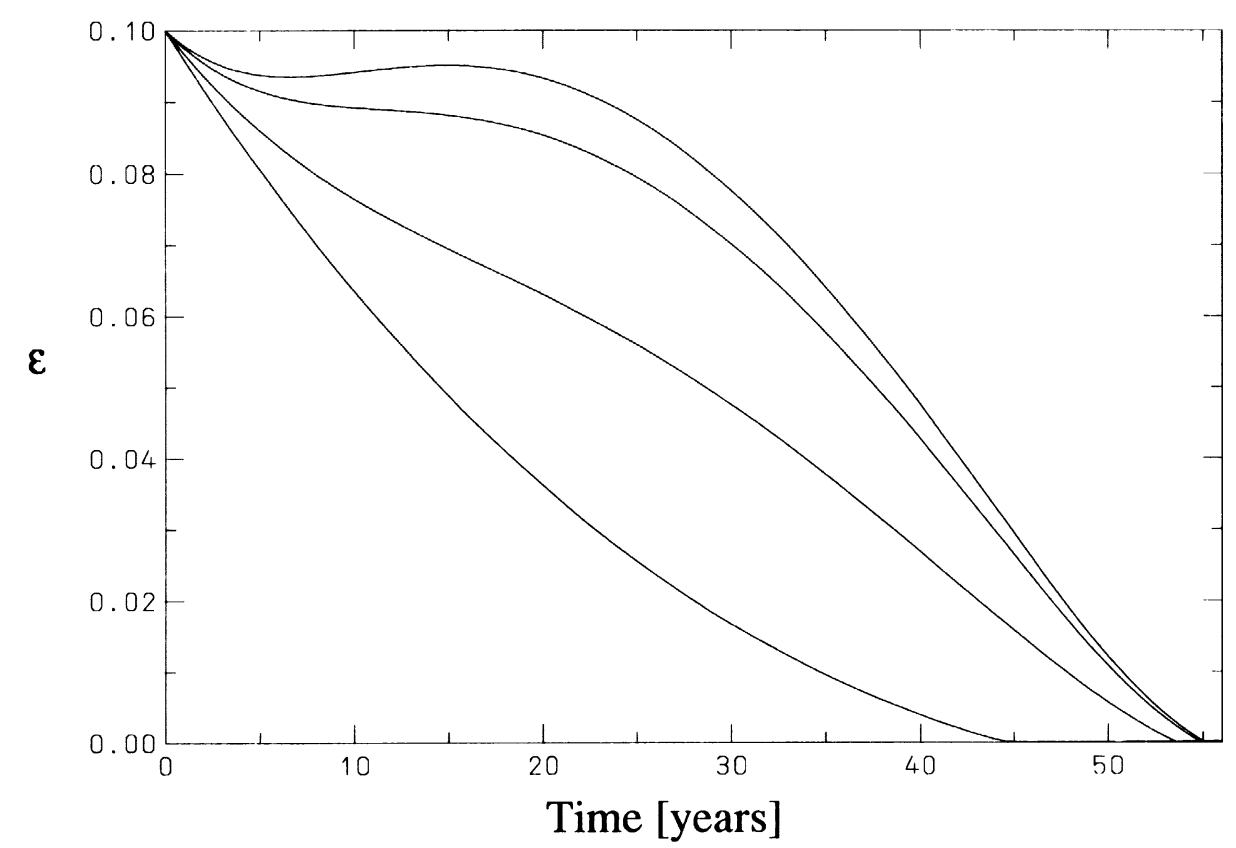

Fig. 2. The change of the eccentricity with increasing inclination for a dust grain of $10 \mu \mathrm{m}$ size. From bottom to top: $i=1^{\circ}, 30^{\circ}, 60^{\circ}, 89^{\circ}$. The other initial elements are in all cases the same: semimajor axis $a=0.1 \mathrm{AU}$, eccentricity $\varepsilon=0.1$, ascending node $\Omega=56^{\circ}$, and the argument of the perihelion $\omega=33^{\circ}$.

can determine the coefficient $\gamma_{\mathrm{H}}=4 \cdot 10^{-10} \mathrm{~cm} \mathrm{~s}^{-2}$ for a spherical dust particle (with radius $s=10 \mu \mathrm{m}$ and density $\left.\rho=1 \mathrm{~g} \mathrm{~cm}^{-3}\right)$.

Comparing the coefficients of the neutral and the plasma Poynting-Robertson force per unit mass, one can determine the distance at which both forces are equal in magnitude:

$$
\frac{\gamma_{\mathrm{H}}}{\gamma_{\mathrm{sw}}}=\frac{n_{\mathrm{H}} v_{\mathrm{H}}^{2}}{n_{\mathrm{sw}}(r) v_{\mathrm{sw}^{2}}}=1
$$

with $n_{\mathrm{sw}}(r)=n_{0}\left(r_{0}^{2} / r^{2}\right)$, where $r_{0}=1 \mathrm{AU}$ is a normalization, one would end at

$$
r \approx r_{0} \sqrt{\frac{n_{0} v_{\mathrm{sw}}^{2}}{n_{\mathrm{H}} v_{\mathrm{H}}^{2}}} \approx 200 \mathrm{AU}
$$

where both forces are almost equal. (Remark: $\vec{v}_{\mathrm{sw}}, \vec{v}_{\mathrm{H}}$ has to be replaced by the respective relative velocities, which lead to a correction of about $\pm 15 \%$ ).

The above analysis holds as long as the orbits of the dust grains do not intersect the termination shock, because the post-shocked plasma is much denser. Moreover, the Mach number is small $(M \leq 0.2)$ and hence the drag coefficient becomes more complicated (Kausch and Fahr, 1997).

Here, as in the case of the near solar wind, we have to calculate the orbital average numerically, thus there is no approximation in the mathematical description of the relative velocity in order to find analytical solutions (Banaszkiewicz et al., 1994). The evolution of a test particle is shown in Figs. 3 and 4. Figure 3 shows the evolution of a dust grain of $10 \mu \mathrm{m}$ size at $20 \mathrm{AU}$, including the interaction of the plasma and electromagnetic Poynting-Robertson effect, as well as the secular perturbation of the four major planets with fixed elements. To demonstrate the power of the neutral gas drag we also calculated the evolution of the dust particle with the same initial conditions, but without the neutral gas drag, which is the dotted line in Fig. 3. There may be differences integrating the equation of motion for the entire system, but to demonstrate the effect for small dust particles even at $20 \mathrm{AU}$ the above secular integration method is sufficient. In Fig. 4 we show the behaviour of a much larger particle $s=1000 \mu \mathrm{m}$ at $45 \mathrm{AU}$ with and without the neutral gas drag. There are small difference in the evolution, but the dynamics of the dust particle is mainly influenced by the interaction of the planets. Because of the lack of space, we can here only show these two examples. Moreover, for other initial condition of the orbits of dust grains, we found the similar behaviour, namely, that the eccentricity increases towards one in a very short time. In a forthcoming publication, we will discuss the neutral gas force in more detail (Scherer, 1998).

The particular feature in Fig. 3 where the semimajor axis increases at about $2.5 \times 10^{5}$ years maybe caused by a close encounter with Saturn. But on the other hand, at this perihelion distance $(\ll 10 \mathrm{AU})$ of a dust grain, our simple assumption of a constant neutral gas density does not longer hold. Moreover, the evolution of dust particles with large eccentricities penetrating deeply into the solar system using an averaging procedure can lead to errors in the evolution of the orbital elements. On the other hand, the error should be small, because the dust grain spends much more time in the orbit near aphelion, especially with large eccentricities, than it does at perihelion. Hence the conclusion, that the orbit of a dust particle becomes parabolic still remains.

All dust grains which are much smaller than $s \ll 1000 \mu \mathrm{m}$ are expelled from the Edgeworth-Kuiper belt on time scales about half a million years, and either fall into the Sun or leave the solar system. Large meteoroids $s \geq 1000 \mu \mathrm{m}$ can survive, at least a couple of millions of years on orbits 


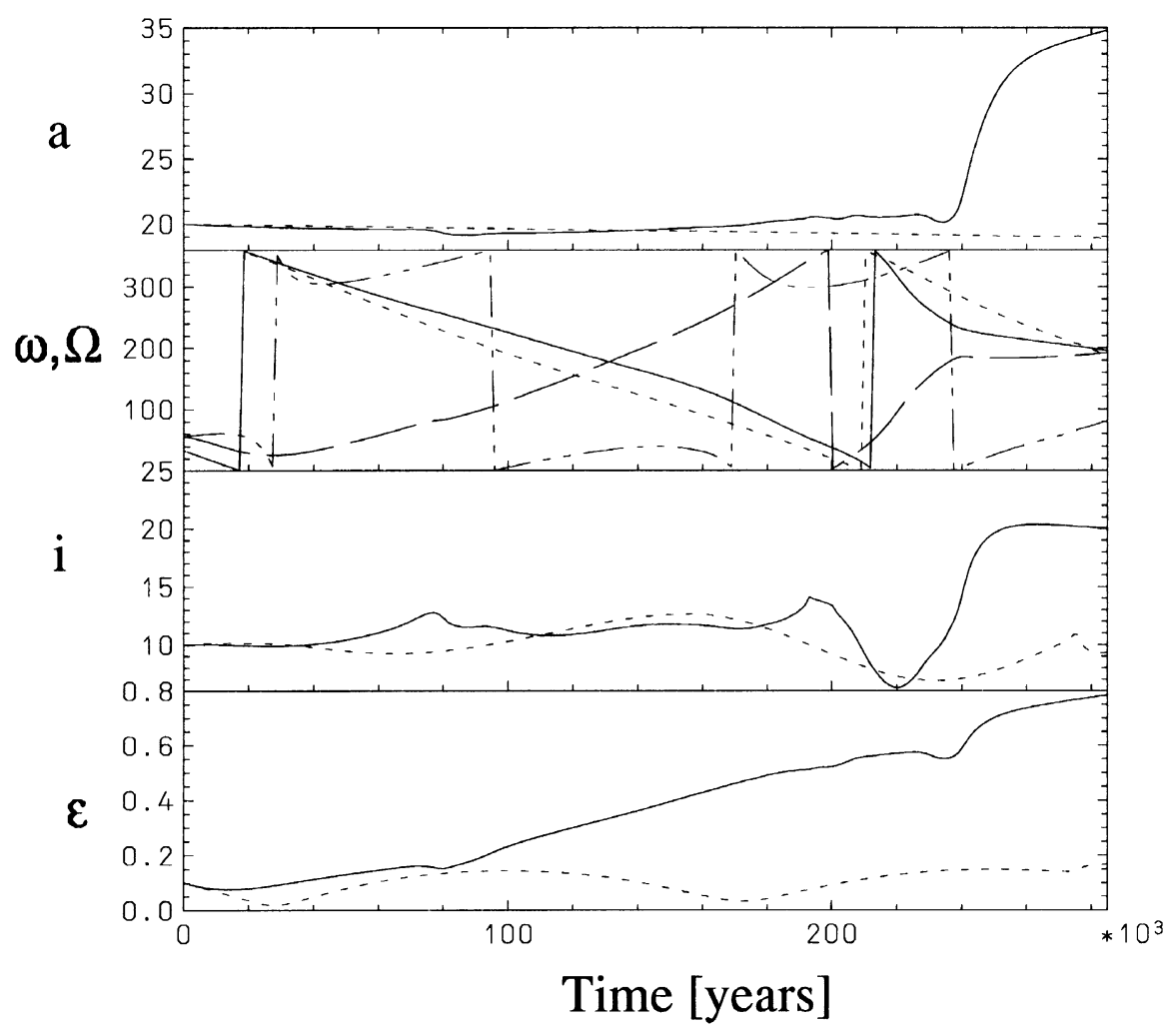

Fig. 3. The perturbation of a test particle at $a=20 \mathrm{AU}$ and $i=10^{\circ}$. The other parameters are the same as in Fig. 2. The solid line includes the neutral gas drag, while the dotted line is without the neutral gas drag. In the second panel from top the solid and dotted line correspond to the ascending node, while the dashed and dashed dotted corresponds to the argument of the perihelion (with and without the neutral gas drag).

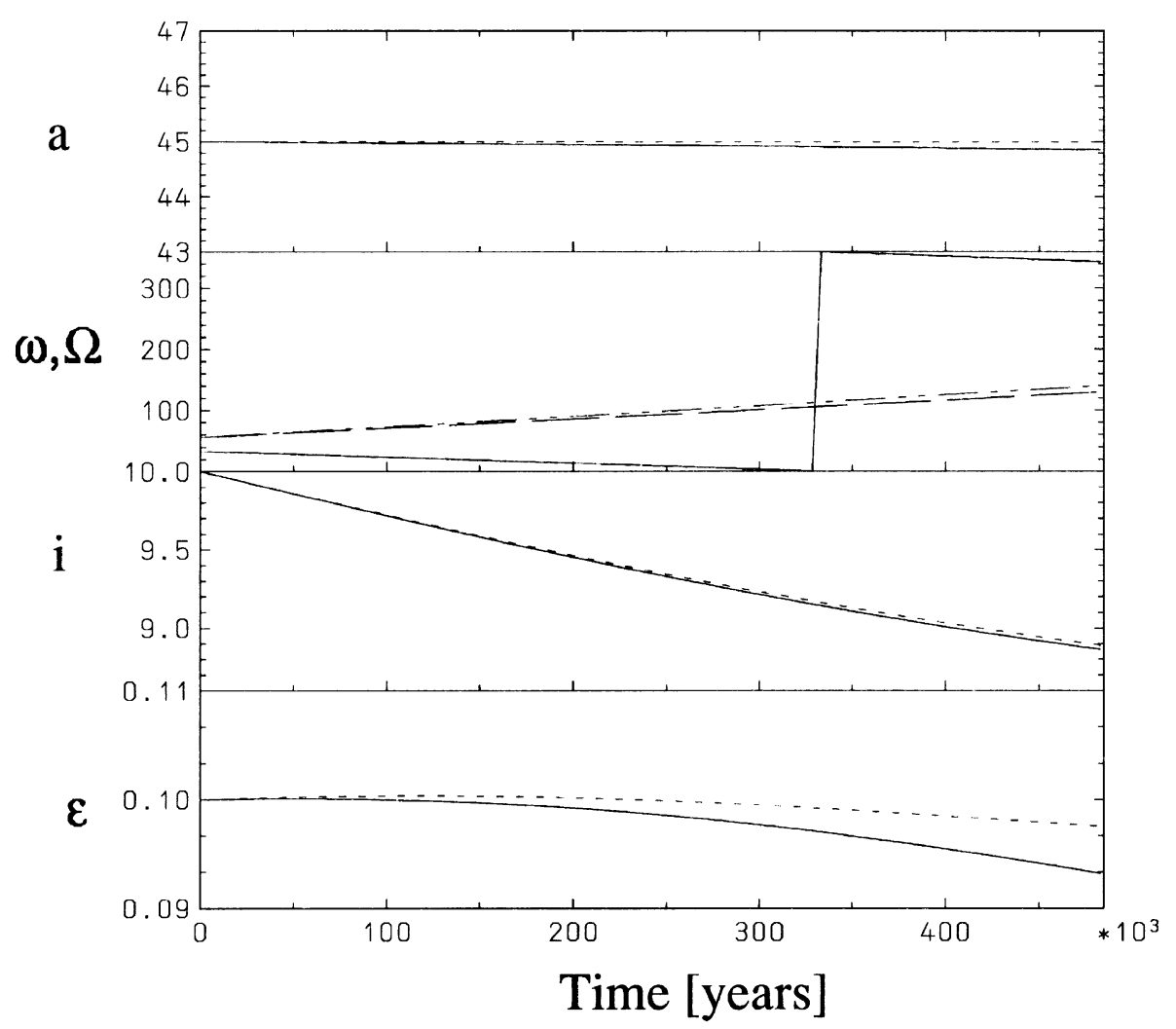

Fig. 4. The same as in Fig. 3 for a dust grain of size $s=1000 \mu \mathrm{m}$ at $a=45$ AU. 
with semimajor axis $a \approx 45$ AU. For much larger semimajor axis the planetary perturbation becomes weaker, and the meteoroids are also swept out by the neutral gas drag.

\section{Conclusion}

We have discussed the plasma Poynting-Robertson force for different regions of the heliosphere: i.e. the near Sun region and the Edgeworth-Kuiper Belt, continuing our earlier studies (Banaszkiewicz et al., 1994; Fahr et al., 1995) inbetween the above regimes, the distant solar wind. In the region near the Sun we used the same formalism as for the distant solar wind, but had to change the plasma model and the drag coefficient. These changes introduced a lot of complications, not only because of the low Mach numbers, and hence a complicated drag coefficient, but also because the solar wind itself has a complicated three dimensional structure. The dynamical behaviour of the dust grains differs from that of the distant solar wind: the eccentricity is no longer a monotonically decreasing function, and also the semimajor axis changes the slope. But further studies are needed, before we can come to firm conclusions on the dynamics of dust near the Sun and to derive a density distribution which is consistent with the Zodiacal light observations.

The situation is different in the Edgeworth-Kuiper Belt. Here we described the drag introduced by the neutral gas flowing into the heliosphere. This mono-directional flow forces the eccentricities of small dust grains to grow rapidly. After almost half a million years the eccentricity approaches one and the dust particle either leaves the solar system or falls into the Sun. Thus the neutral gas drag dominates the evolution of the dust particles in the Edgeworth-Kuiper Belt, especially during the passage of the Sun through molecular clouds, or even, when the Sun enters a spiral arm of the galaxy. The low number density of the interstellar medium in the last million years seems to be a more extraordinary situation. The interstellar number density outside the local fluff is higher and hence the migration of Edgeworth-Kuiper Belt dust to high eccentricities and inclinations on a long-time average takes place on much shorter time scales. Therefore the sources of dust grains in the Edgeworth-Kuiper disk are most likely due to collisions, erosion of larger bodies due to high speed impacts of interstellar grains, or sputtering by the solar wind. In the future, we will study the Ulysses dust data, and try to identify and determine the Edgeworth-Kuiper Belt dust and to get some hints on the dust production rate in the Belt.

\section{References}

Banaszkiewicz, M., H. J. Fahr, and K. Scherer, Evolution of dust particle orbits under the influence of solar wind outflow asymmetries and the formation of the zodiacal dust cloud, Icarus, 107, 358, 1994.

Bougeret, J. L., J. H. King, and R. Schwenn, Solar radio burst and in-situ determination of interplanetary electron density, Sol. Physics, 90, 401$411,1984$.

Bzowski, M., H.-J. Fahr, D. Rucinski, and H. Scherer, variation of neutral hydrogen moments in the heliosphere during the solar cycle, Astron. Astrophys., 1997 (in press).

Fahr, H.-J., The interstellar gas flow through the heliospheric interface region, Space Sci. Rev., 78, 199-212, 1996.

Fahr, H.-J., K. Scherer, and M. Banaszkiewicz, The evolution of the zodiacal dust cloud under plasma drag and Lorentz forces in the latitudinally asymmetric solar wind, Planet. Space Sci., 43, 301, 1995.

Fahr, H.-J., H. Fichtner, and H. Scherer, Observation of EUV resonance emissions of pick-up ions close to the solar corona, submitted to Astron. Astrophys., 1998.

Frisch, P. C., Characteristics of nearby interstellar matter, Space Sci. Rev., 72, 499-592, 1995.

Geiss, J. and M. Witte, Properties of the interstellar gas inside the heliosphere, Space Sci. Rev., 78, 229-238, 1996.

Gor'kavyi, N. N., L. M. Ozernoy, J. C. Mather, and T. Taidakova, Quasistationary states of dust flows under Poynting-Roberston drag: New analytical and numerical solutions, Astrophys. J., 488, 268, 1997.

Kausch, T. and H. J. Fahr, Interstellar gas filtration to the inner heliosphere under the self-consistent influence of a pick-up ion modulated termination shock, Astron. Astrophys., 325, 828-838, 1997.

Muhleman, D. O. and J. D. Anderson, Solar wind electron densities from Viking dual-frequency radio measurements, Astrophys. J., 247, 1093$1101,1981$.

Northrop, T. G., Dusty plasmas, Physica Scripta, 45, 475-490, 1992.

Northrop, T. G. and T. J. Birmingham, Plsama Drag on a dust grain due to Coulomb collisions, Planet. Space Sci., 38, 319-326, 1990.

Osterbart, R. and H.-J. Fahr, A Boltzmann-kinetic approach to describe the entrance of neutral interstellar hydrogen into the heliosphere, Astron. Astrophys., 264, 260-269, 1992.

Rucinski, D. and M. Bzowski, Modulation of interplanetary hydrogen density distribution during the solar cycle, Astron. Astrophys., 296, 248, 1995.

Rucinski, D., A. C. Cummings, G. Gloeckler, A. J. Lazarus, E. Möbius, and M. Witte, Ionization processes in the heliosphere-Rates and methods of their determination, Space Sci. Rev., 78, 73, 1996.

Scherer, K., Drag forces on interplanetary dust grains induced by the neutral gas, Icarus, 1998 (submitted).

Scherer, H., H.-J. Fahr, and J. T. Clarke, Refined analysis of interplanetary HLyman-Alpha spectra obtained with the Hubble-Space Telescope GHRS spectrometer, Astron. Astrophys., 325, 745-754, 1997.

Scherer, K., I. Mann, and G. Reaves, On the dynamics of the zodiacal dust cloud near the Sun, in NASA Conference Publication 3343: From Stardust to Planetsimals: Contributed Papers, edited by M. E. Kress, A. G. G. M. Tielens, and Y. J. Pendleton, pp. 209-212, 1996.

K. Scherer (e-mail: scherer@lindust.mpae.gwdg.de) and H.-J. Fahr 